\title{
Endoscopic esophagogastric anastomosis with luminal apposition Axios stent (LAS) approach: a new concept for hybrid "Lewis Santy"
}

\section{다(1) $(9)$}

\author{
Authors \\ Adrian Culetto ${ }^{1,2}$, Jean-Michel Gonzalez ${ }^{1,2}$, Geoffroy Vanbiervliet ${ }^{2,3}$, Pablo Miranda Garciaa, , Juan Ignacio \\ Tellechea $^{1,2}$, Emmanuelle Garnier ${ }^{2}$, Stephane Berdah ${ }^{2,4}$, Marc Barthet ${ }^{1,2}$
}

Institutions

1 Department of Gastroenterology, Public Assistance Hospitals of Marseille, North Hospital, Marseille, France

2 Aix-Marseille University, CERC, Faculty of Medecin, Marseille, France

3 Department of Endoscopy, University Hospital of Nice, Nice, France

4 Department of Digestive Surgery, Public Assistance Hospitals of Marseille, Marseille, France

submitted 26.11.2016

accepted after revision: 10.2.2017

Bibliography

DOI https://doi.org/10.1055/s-0043-106577 |

Endoscopy International Open 2017; 05: E455-E462

(c) Georg Thieme Verlag KG Stuttgart · New York

ISSN 2364-3722

Corresponding author

Adrian Culetto, MD, Department of Gastroenterology, Public Assistance Hospitals of Marseille, North Hospital, Marseille, France

culetto.a@chu-toulouse.fr

\section{ABSTRACT}

Background and study aims Esophagogastric anastomosis (EGA) has a high risk of leakage. Based upon our experience in endoscopic gastrojejunal anastomosis using LAS, the aim of this study was to verify the technical feasibility and the safety of performing an EGA using a hybrid approach (endoscopic and surgical).

Materials and methods A pilot prospective study was performed on 8 survival pigs. The procedure was carried out in 2 stages: (i) surgical step consisting of an esogastrectomy by laparotomy with separated suture of the esophagus and stomach; (ii) endoscopic esophagogastric anastomosis using the LAS. The first 2 pigs allowed for the setting of the 2 steps procedure, and 6 were included in the study for assessing the efficacy and safety of the procedure with a 3-week survival course. The primary endpoint was morbidity and mortality.

Results All procedures were successfull. The mean operative time was 98 minutes, with a mean endoscopic time of 46 minutes. Three early deaths occurred within the first weeks, unrelated to the LAS anastomosis. At 3 weeks, endoscopic assessment followed by necropsy demonstrated the right position and the endoscopic removability of the stent with good patency of the esophagogastric anastomosis, without leakage of the endoscopic suture. Pathological examination confirmed the patency of the anastomosis with fusion of mucosal and muscle layers.

Conclusion Endoscopic esophagogastric anastomosis with LAS is feasible and reproducible, without anastomotic leakage. It could be a new alternative to perform safe anastomoses, as part of a hybrid approach (surgical and endoscopic).

\section{Introduction}

Surgical resection of the esophagus is usually indicated for cancer, Barrett's esophagus or benign conditions including stricture, diseases such as achalasia, paraesophageal hernia, and other complex esophageal disorders after endoscopic failure. More than $85 \%$ of such procedures are dedicated to resection of esophageal tumors. Esophageal resection has justifiably earned a reputation for high mortality, morbidity (ranging from $30 \%-60 \%$ and $8 \%-23 \%$, respectively), and poor quality of life [1]. Surgical approaches are based upon transabdominal and/or thoracic accesses, using either thoracotomy or thoracoscopy, with development of minimally invasive techniques such as Lewis Santy surgery [2]. That procedure has significant morbidity, often associated with the occurrence of anastomotic leakages $(9 \%-12 \%$ of cases) $[3,4]$.

Our team demonstrated previously that it was possible to perform safe and effective endoscopic gastrojejunal anastomosis using luminal apposition stent (LAS) [5]. Thus, we used this self-expandable fully-covered stent (Cold Axios, Boston Scienti- 
fic, USA) specifically designed with both flanges diameter twice that of the "waist" section, in order to propose a new type of endoluminal anastomosis [6].

Our hypothesis was that a hybrid approach combining minimally invasive esophagectomy and endoluminal insertion of LAS would be less morbid and less cumbersome for creating a safe and functional esophagogastric anastomosis. That could add another a minimally invasive procedure to the surgeons' and endoscopists' armamentarium.

The aim of our study was to document the technical feasibility and safety of achieving an esophagogastric anastomosis using the hydrid approach (endoscopic and surgical) with LAS method. Feasibility and efficacy were assessed using technical parameters such as procedure time, technical difficulty, and the occurrence of intraoperative adverse events. Safety was assessed by monitoring the animals postoperatively for any clinical signs of intra-abdominal infection and sepsis. Anastomotic integrity and patency were evaluated grossly during necropsy as well as histologically.

\section{Materials and methods}

\section{Study design}

This was a prospective experimental survival animal study conducted at the Center for Surgical Education and Research (CERC) of the Faculty of Medicine, Aix-Marseille University. Institutional review board approval was achieved prior to conducting the study.

The experimental protocol consisted of performing a surgical and endoscopic procedure under general anesthesia on 8 consecutive healthy, young domestic female Yorkshire "minipigs," aged of 3 to 4 months and weighing between 28 and $34 \mathrm{~kg}$. The first 2 pigs allowed for setting up the different steps in the procedure and its technical feasibility, and the 6 other animals were included in the current study. Euthanasia and necropsy were performed following a 3-week survival period.

\section{Preoperative animal management}

All animals were kept fasting for 24 hours prior to intervention. Anesthesia was induced with intramuscular injection of $120 \mathrm{mg}$ of azaperone (Stresnil) coupled with $70 \mathrm{mg}$ of ketamine, followed by endotracheal intubation. Then, anesthesia was maintained by continuous intravenous infusion of propofol at a rate of $100 \mathrm{mg}$ per hour (Diprivan 2\%), and fentanyl was given at a dose of $100 \mathrm{mcg}$ per hour for analgesia, with monitoring of heart rate and oxygen saturation via pulse oximetry. The animals also received intraoperatively $1 \mathrm{~g}$ of cefotaxime as antibiotic prophylaxis. All animals were placed in supine position for the procedure, and abdominal disinfection was carried out using betadine before initiating the laparotomy.

\section{Endoscopic and surgical equipment}

For the surgical part, we used standard sterilized surgical equipment including linear staplers and a bipolar coagulation device. Two gastrointestinal surgeons performed this step. The endoscopic part was performed by 3 interventional endoscopists using a double-channel video gastroscope (3.8- and

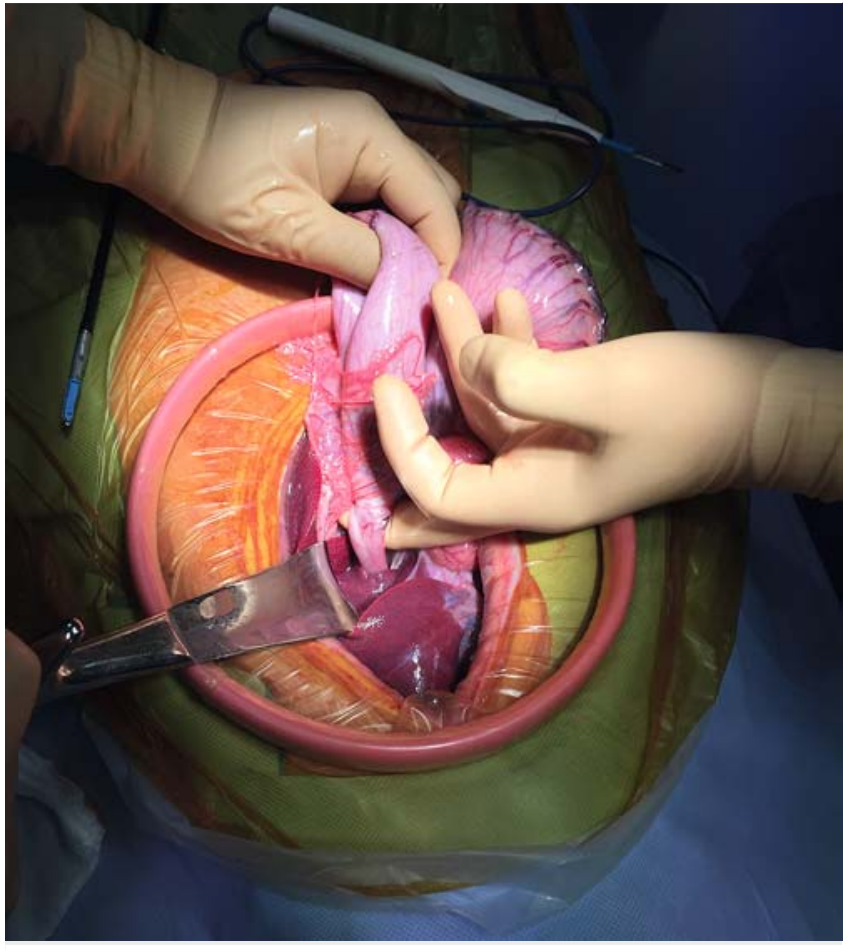

Fig. 1 Resection of the lower esophagus 2 to $3 \mathrm{~cm}$ above the cardia using a linear stapler.

2.8-mm channel diameters; Olympus Corporation, Tokyo, Japan) for creating the gastroesophageal anastomosis. The electrosurgical unit used was the Olympus ESG-100 (Olympus Corporation, Tokyo, Japan). The sterilized endoscopy equipment that helped completing the procedure (forceps, needle, knife, guide wire, catheter and stents) was disposable. The luminal apposition stent (Cold Axios, Boston Scientific, USA) is fully covered, $10 \mathrm{~mm}$ long between the flanges and $15 \mathrm{~mm}$ in diameter, braided nitinol, with bilateral anchor flanges of $24 \mathrm{~mm}$ in diameter. The stent was deployed through a $10.5 \mathrm{Fr}$ catheter.

\section{Procedural steps}

The surgical step started with a midline laparotomy by carrying out a cold knife incision and an opening plane by plane, using electrocautery. Abdominal dissection was conducted allowing release of the stomach and mobilization of the gastroesophageal junction. Then, if the diaphragmatic hiatus was not large enough for ascent of the stomach, it was widened by sectioning of the diaphragm pillar. First the surgeon resected the lower esophagus 2 to $3 \mathrm{~cm}$ above the cardia and used a linear stapler ( $\triangleright$ Fig. 1). Resection of the upper pole of the stomach was then completed and tunneling of the stomach's body was performed using a linear stapler.

After introduction of the scope and cleansing of the esophagus, the esophagus was opened just above the surgical suture line using endoscopy. That access was realized initially by making a plane by plane full-thickness esophageal incision with a Hook Knife (Olympus Corporation, Tokyo, Japan). However, for safety reasons (pleural wounds with the knife), the knife was then replaced by a puncture with a 19 G needle (Cook medical 


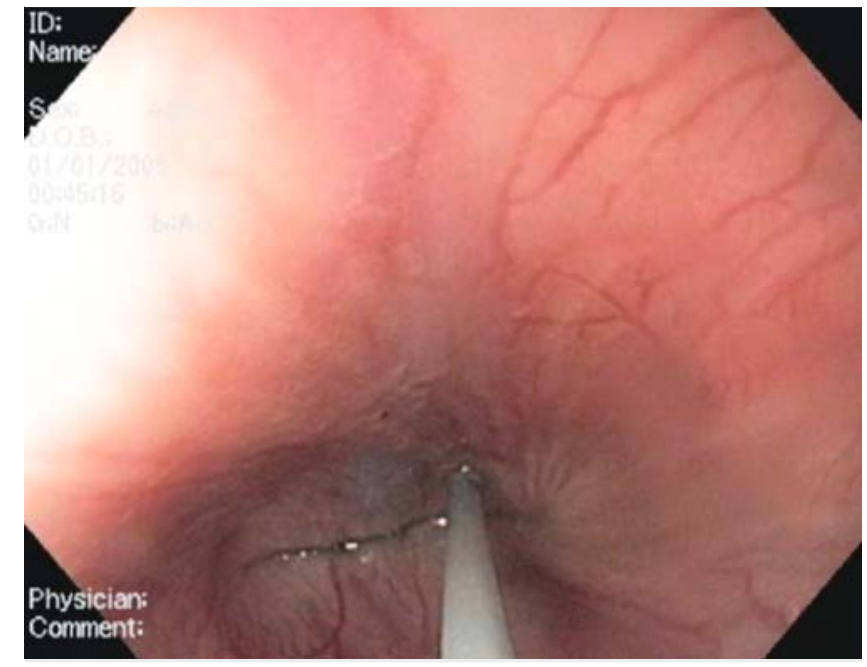

- Fig. 2 Puncture with a $19 \mathrm{G}$ needle(Cook medical in Limerick, Ireland) above the aureastomosis esophagus.

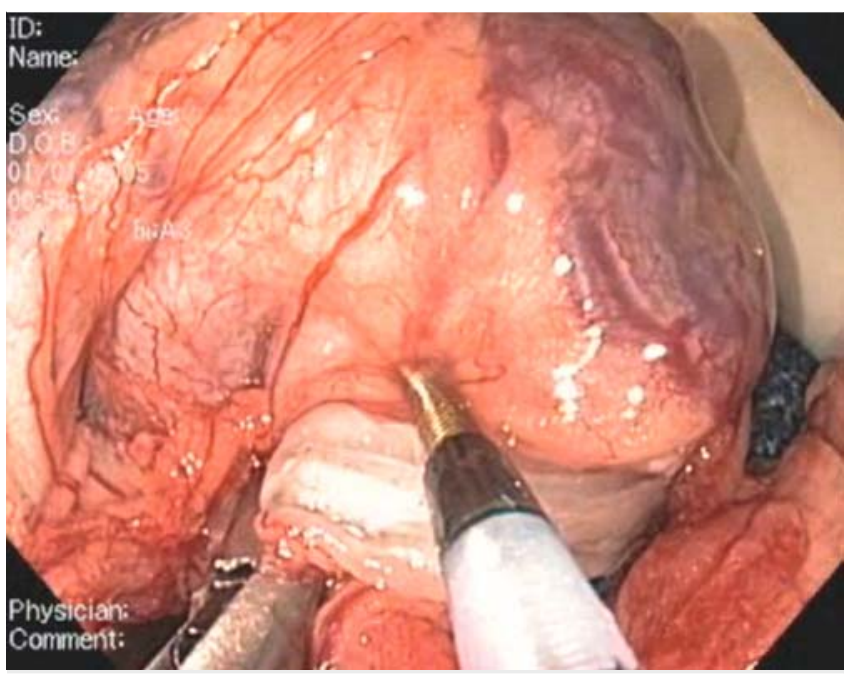

- Fig. 3 Access to the gastric lumen at the upper edge of the gastric suture using a cystostome $10 \mathrm{Fr}$ (Cook Medical, Limerick, Ireland) by a section of current Pulse Cut Fast $80 \mathrm{~W}$.

in Limerick, Ireland) ( $\triangleright$ Fig. 2). That enabled insertion of a guide wire (Jagwire Stiff, Boston Scientific Corporation, Natick, USA) and hydraulic dilation up to $18 \mathrm{~mm}(15-18 \mathrm{~mm}$ balloon CRE, Boston Scientific Corporation, Natick, USA). As a result, passage of the endoscope through the esophageal wall and access to the mediastinum and abdominal cavity were enabled.

Once the stomach was observed, it was grasped using forceps introduced through one of the operating channels (Twin Grasper; OVESCO AG, Tuebingen, Germany) so as to stabilize through the end of the procedure. Access to the gastric lumen at the upper edge of the gastric stapler line was achieved using a $10 \mathrm{Fr}$ cystostome (Cook Medical, Limerick, Ireland) and applying a Pulse Cut Fast current $(80 \mathrm{~W})(\triangleright$ Fig. 3). Once the stomach cavity was reached, the tip of the cystostome was removed, a superstiff guidewire was advanced instead, and the cystostome was exchanged with the catheter of the Axios stent.

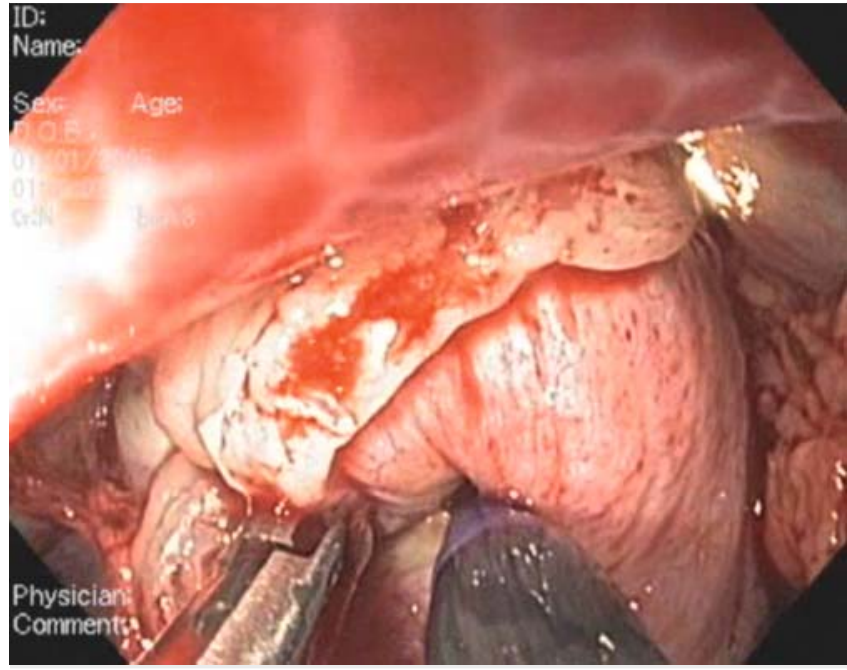

Fig. 4 With delivery of the catheter from the stent to the gastric position, expansion of the distal frange of the stent in the gastric cavity could be visualized.

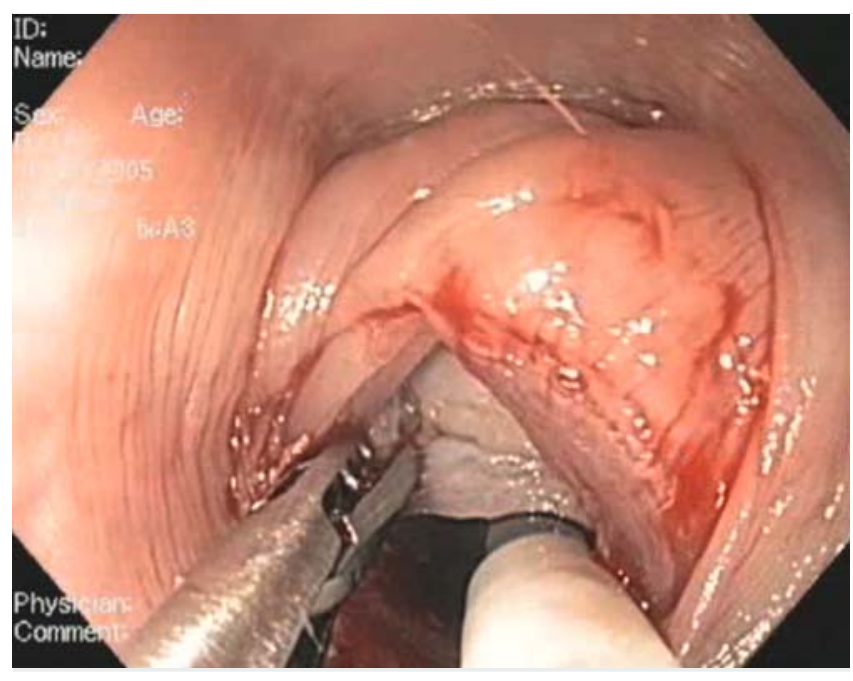

- Fig. 5 Pulling from the stomach into the mediastinum was achieved by using the endoscope to guide the catheter of the stent and also the Twin Grasper, plus assistance in the abdomen from a surgical aid.

When the delivery catheter was in the gastric position, we then expanded the distal frange of the stent into the gastric cavity ( $\triangleright$ Fig.4). Then, using both forceps and stent catheter (with the stent half deployed), we pulled the stomach back towards the esophagus through the mediastinum by gently removing the scope. This phase was also facilitated by help from the surgeons in the abdominal cavity ( $\mathbf{F i g} . \mathbf{5}$ ). Once the correct position in the esophageal lumen was achieved, with the stomach in contact with the esophagus in the mediastinum, we released the Twin Grasper forceps and completed stent placement by deploying its proximal flange ( $\triangleright$ Fig. $\mathbf{6}$ ). Correct positioning of the stent was confirmed by the presence of gastric juice in the stent ( $\triangleright$ Fig. 7). Finally, the abdominal incision was manually sutured at the end of the procedure. 


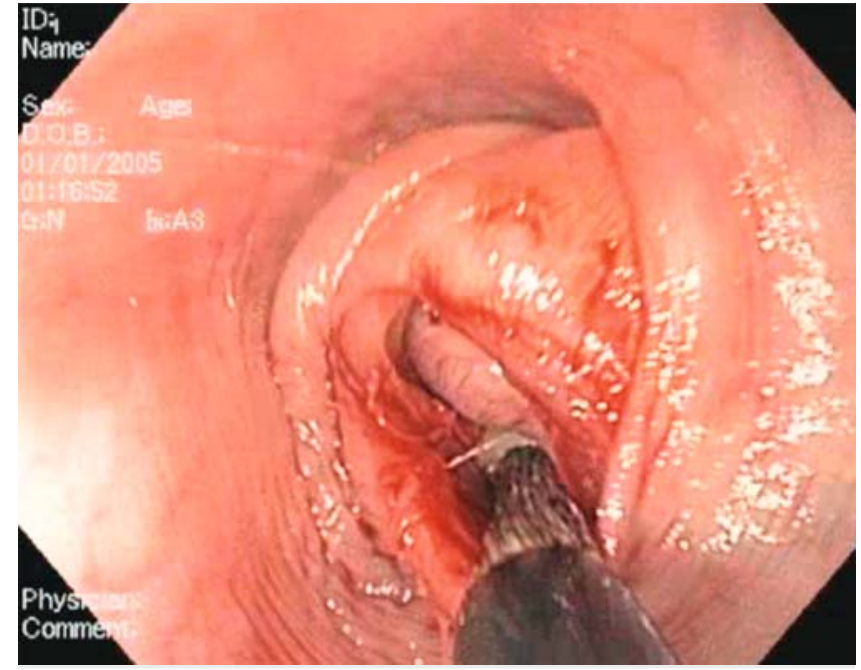

- Fig. 6 The proximal flange was deployed with correct positioning of the gastric cavity in the mediastinum and in contact with the esophagus had been confirmed.

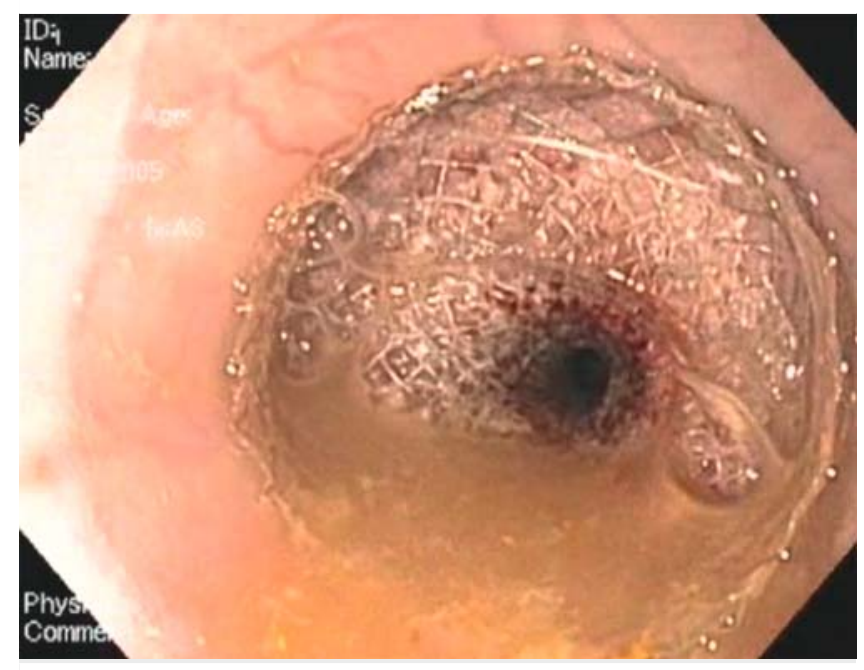

- Fig. 7 Correct positioning of the stent was confirmed by the appearance of gastric juice in the stent.

\section{Follow-up and post-operative protocol}

The first 2 animals were euthanized at the end of the procedure to assess the technical feasibility of the procedure and check for correct stent position. Six other animals were clinically observed for a period of 3 weeks.

After recovery from anesthesia, the animals were kept fasting with access to water until the third postoperative day. Daily antibiotic prophylaxis (cefotaxim $1 \mathrm{~g}$ ) was administered intramuscularly for 7 days. Institution of nutrition was performed gradually: a quarter of the usual food ration was given for 48 hours, then half portions for 48 hours before normal feeding from postoperative Days 3 to 21. Clinical follow-up was performed twice daily (monitoring of overall behavior, pain, food intake, fever, and bowel and urinary function).

\section{Euthanasia and histological assessment}

The euthanasia was realized after 21 survival days by lethal injection of potassium chloride to animals under general anesthesia. In the case of death during the 3-week period, necropsies were performed to determine whether signs of anastomotic leakage or peritonitis were present. Before euthanasia, upper endoscopy was performed to check for correct position, permeability and crossing of the stent and to remove the stent in order to analyze the anastomosis. The peritoneal cavity and mediastinum were then inspected after laparotomy and sternotomy for signs of peritonitis, and all organs were macroscopically examined for signs of infection, scar formation, and necrosis. Finally the entire anastomosis was removed for histopathological examination.

The anastomotic healing pattern was assessed by histological analysis. To assess its integrity, the anastomotic site was also histologically observed for presence of scar formation, necrosis, inflammation and fistula.

\section{Statistical analysis}

Data are presented as mean \pm standard deviation (SD). The small size of the study did not allow for comparative tests in univariate analysis by Fisher's exact test or the use of a chisquared distribution to search for predictive factors of death. Unpaired Student's $t$ tests were used to determine the significance of differences between means. A $P$ value $<0.05$ was considered statistically significant. Analyses were performed using the InStat 3.1a software (GraphPad,La Jolla, CA, USA).

\section{Results}

Data related to technical and clinical outcomes from esophagogastric anastomosis are summarized in > Table 1.

\section{Primary endpoint: feasibility of the procedure}

Esophagogastric anastomosis with LAS was successfully performed in 8 animals, with excellent reproducibility. Mean operative time for the entire procedure was 98 minutes (range 64-150), with mean endoscopic time of 46 minutes (range 24-70). Half the endoscopic times were 45 minutes or less. We also observed a learning curve effect with a gradual improvement in endoscopic and surgical times.

In 2 of 8 procedures, it was necessary to use two Axios stents, due to avulsion of the distal flange when pulling back the stomach into the mediastinum. This was subsequently avoided in the last procedures by tunneling the stomach, enlarging the diaphragmatic hiatus, facilitating (surgeons) the gastric ascension and not pulling back only with the catheter of the stent.

\section{Intraoperative adverse events}

During anesthesia, 1 animal developed a significant hypoxemia $\left(\mathrm{SatO}^{2}<90 \%\right)$. That animal died during the recovery phase immediately after surgery following major hypoxemia and bradycardia. That event was imputable to major pneumothorax due to a wound of the pleura, which was related to opening of the 
- Table 1 Summary of technical and clinical outcomes from esophagogastric anastomosis with luminal apposition stent (LAS) by hydrid approach in 8 pigs.

\begin{tabular}{|c|c|c|c|c|c|c|c|c|}
\hline & \multicolumn{8}{|c|}{ Animal no. } \\
\hline & 1 & 2 & 3 & 4 & 5 & 6 & 7 & 8 \\
\hline \multicolumn{9}{|l|}{ Anesthesia } \\
\hline - Oxygen rate $<90 \%$ & No & No & No & No & No & Yes & No & No \\
\hline - Tachycardia>120/min & No & No & No & No & No & No & No & No \\
\hline - Total time, minutes & 150 & 110 & 85 & 114 & 114 & 64 & 76 & 68 \\
\hline \multicolumn{9}{|l|}{ Surgical procedure } \\
\hline - Total time, minutes & 75 & 55 & 45 & 38 & 39 & 28 & 25 & 28 \\
\hline - Size esophagus resection, cm & 2 & 1.5 & 2 & 1.5 & 1.5 & 2 & 2 & 2 \\
\hline - Size stomach resection, $\mathrm{cm}$ & 10 & 8 & 13 & 9 & 7 & 11 & 15 & 11 \\
\hline \multicolumn{9}{|l|}{ Endoscopic procedure } \\
\hline - Total time, minutes & 70 & 45 & 35 & 61 & 60 & 24 & 41 & 35 \\
\hline - Height line esophagus staples, cm & 54 & 52 & 50 & 48 & 60 & 50 & 52 & 52 \\
\hline \multicolumn{9}{|l|}{ Follow-up } \\
\hline - Transit & & & Yes & Yes & Yes & N & Yes & Yes \\
\hline - Weight before procedure, kg & & & 30.3 & 31.4 & 34 & 28.8 & 31.6 & 32.7 \\
\hline - Weight after procedure, kg & & & 23.9 & 25.2 & $\mathrm{~N}$ & N & 26.9 & N \\
\hline - Occlusion & & & No & No & No & $\mathrm{N}$ & No & No \\
\hline - Sepsis, postoperative day & & & No & No & No & N & No & Yes (10) \\
\hline - Death, postoperative day & & & No & No & Yes (2) & Yes $(0)$ & No & Yes (10) \\
\hline \multicolumn{9}{|l|}{ Autopsy } \\
\hline - Peritonitis signs & & & No & No & No & No & No & Yes \\
\hline - Anastomotic leakage & & & No & No & No & No & No & No \\
\hline - Local inflammation & & & Yes & No & No & No & Yes & Yes \\
\hline - Right position stent & & & Yes & Yes & Yes & Yes & Yes & No data \\
\hline - Endoscopic stent removability & & & Yes & Yes & Yes & Yes & Yes & No data \\
\hline
\end{tabular}

esophagus with the Hook-Knife. After that event and for safety reasons, we decided to change our access technique and open the esophagus by performing a needle puncture followed by 18-mm hydraulic balloon dilation over a guide wire.

\section{Postoperative outcome and follow-up}

In total, 5 animals were completely followed. Two animals died prematurely without evidence of anastomotic dysfunction during postmortem endoscopy. For the first animal, the death occurred 48 hours after surgery. During its necropsy, no complications of the anastomosis or peritonitis were found. The death was attributed to pulmonary embolism, in a context of a long operating time (114 minutes). The second death occurred on the 10th postoperative day due to gastric ischemia. Necropsy revealed peritonitis with necrosis of the stomach. It was attrib- uted to a possible volvulus of the stomach during the surgical phase leading to a gastric ischemia.

Regarding the animals that survived, no signs of peritonitis or sepsis were observed during the 3-week follow-up period. Refeeding was progressive from the third postoperative day. However, a normal daily intake ration could not be reached due to a reduction in gastric volume and probable section of the vagus nerve during the diaphragm hiatus enlargement. Weight loss over 3 weeks was evaluated at $5.7 \mathrm{~kg}$ on average (range $4.7-6.4 \mathrm{~kg}$ ). In 2 animals, acid reflux (presence of foam in the mouth) likely was related to the vagus nerve lesion and to loss of the lower esophagus sphincter. 


\section{Results at 3 weeks}

Endoscopic evaluation was realized for the 3 animals surviving at the end of follow-up. Correct positioning of the stent and its crossing with the double working channel endoscope (without dilatation) were confirmed in all those cases. There was no gastric or esophageal endoscopic lesion related to the stent. The removal rate for stents was $100 \%$, using a rat tooth forceps, and all were removed without any difficulty. Endoscopic evaluation after stent withdrawal confirmed the excellent quality of the anastomoses. In only 1 animal, a fistula was identified, but it was covered by the proximal flange on the line of the esophageal surgical sutures ( $\bullet$ Fig. 8 ) and did not have any clinical consequences.

At necropsy, 1 animal presented with a small abscess on the abdominal suture. In 2 other animals, 2 small abscesses were found on the surgical gastric suture. No cases of fistula or abscess on the endoscopic anastomosis or peritonitis were identified.

\section{Histological analysis}

In all the animals that survived, esophagogastric anastomosis sites were available for histological examination. At the level of the anastomotic esophagogastric junction, we observed complete fusion of mucosal and muscular layers with mild to moderately acute and chronic inflammatory changes. These included highly polymorphic granulomatous tissue with infiltration of lymphocytes, rare plasma cells, macrophages, and neutrophils. The submucosa was infiltrated with collagen, fibroblasts and new blood vessels.

\section{Discussion}

Surgical treatment of the esophagus is indicated for benign or malignant diseases of esophagus, and it remains the primary treatment for local regional esophageal cancer, although its role in superficial (T1a) cancers and squamous cell cancer is evolving. But this treatment has high rates of mortality and morbidity [1]. The main morbidity is related to anastomotic fistula or disunion due to the difficulty of intrathoracic anastomosis. The mortality rate in cases of fistula reaches $36 \%$ and the hospital stay up to 46 days $[7,8]$.

Several techniques are described and usually performed in expert centers. These resections and reconstructions remain technically challenging operations. Complications from these surgeries are demonstrated as directly linked to the number of resections performed by individual surgeons or individual hospital systems. A recent meta-analysis of the volume-outcomes relationship reviewing 27,843 esophageal operations in 9 separate clinical series published since 2000 demonstrated an overall in-hospital mortality rate between $2.8 \%$ and $8.5 \%$ [9]. Surgical teams have expended significant time and effort in trying to demonstrate that one surgical approach has significant advantages over another one. The Ivor Lewis esophagectomy was first described as a completely minimally invasive esophagectomy in 1999. That technique associates transabdominal and transthoracic approaches, in which the anastomosis is being performed

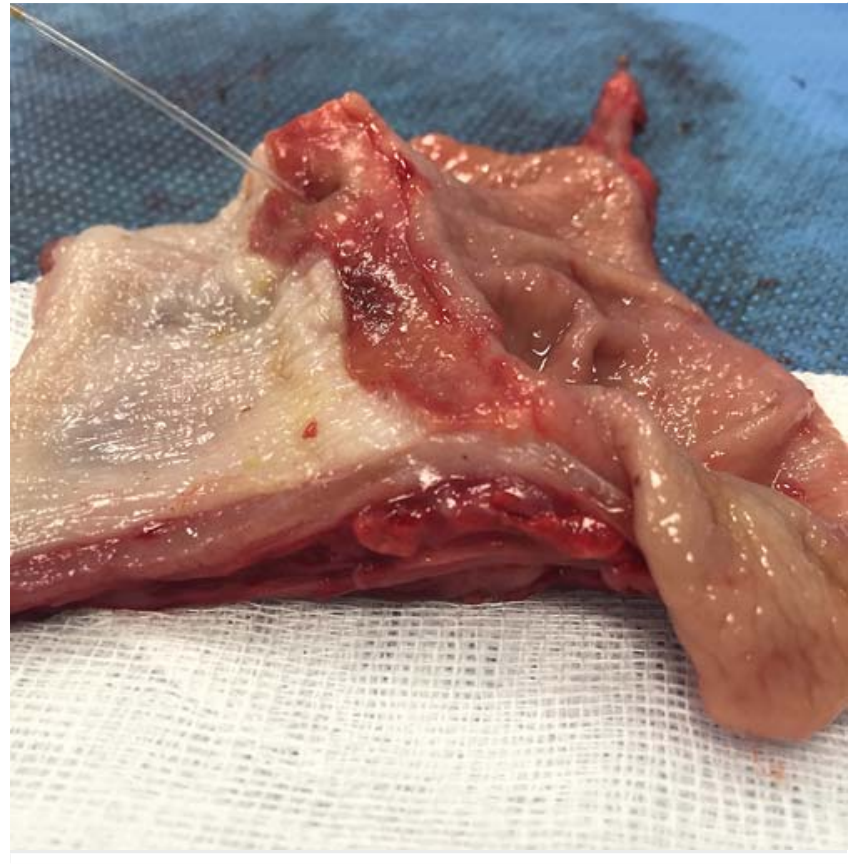

- Fig. 8 The fistula was covered by the proximal flange on the line of esophageal surgical sutures.

within the thoracic cavity. Randomized controlled trials and meta-analysis suggested short-term benefits of minimally invasive esophagectomy comparing to open procedures [10-12]. Despite the dramatically increased application of minimally invasive and hybrid esophageal resection approaches, the procedures are still associated with a non-negligible rate of anastomotic leakages, or parietal and anesthetic complications [1315]. The most common complication is the anastomotic leak or fistula, with a rate ranging from $8 \%$ to $12 \%[3,4]$. This outcome is comparable to the open procedure anastomotic leak rate, which is $9.1 \%$ [16]. Furthermore, the mortality rate in case of fistula reaches $36 \%$ and hospital stay up to 46 days [7, $8,12]$. Multiple risk factors have been identified for such complications, including heart failure, hypertension and renal insufficiency, which are common comorbidities in the targeted population $[13,14]$. Despite the importance of surgical esophageal resection, there is no strong consensus in the literature on the best technique for performing esophagogastric anastomosis [17-21].

Thus, a promising alternative for the execution of minimally invasive anastomosis may be Natural Orifice Transluminal Endoscopic Surgery (NOTES), because it allows mediastinal or peritoneal access for performing surgical procedures without parietal incisions. In 2005 were performed the first NOTES procedures, currently performed on humans, techniques based on a transluminal access for transgastric access. The first endoscopic experimental approach for pure NOTES gastrojejunal anastomosis was recently developed and tested in porcine non-survival and survival experiments [22]. In that regard, we have recently explored the concept of creating an experimental "pure" NOTES gastrojejunal anastomosis in different pig models to achieve a NOTES transgastric bypass [23,24]. These pro- 
cedures using dedicated endoscopic techniques and devices were feasible but result in a huge time-consuming procedure and associated high complication rates. We recently decided to improve our technique of gastrojejunal anastomosis using the concept of the tissue-apposing stent [5]. This stent (Cold Axios, Boston Scientific, USA) is self-expandable and fully covered and designed with 2 collars at the ends, which allow good apposition tissue together and thus a new approach to endoluminal anastomosis [6]. It can be used across the desired anastomotic site to hold tissue layers in apposition. Our results in pure NOTES gastrojejunal anastomosis using this device have shown a constant technical success with a significant reduced mean operative time [5]. No leakage of the anastomosis was observed during follow-up of the 6 specimens. A first human case to pure NOTES gastrojejunal anastomosis was performed successfully without complication [25].

In our series, we confirmed the technical feasibility of achieving esophagogastric anastomosis using a hybrid approach associating surgical and endoscopic techniques (NOTES) in order to improve the anastomotic suture and reduce postoperative complications. Indeed, we did not identify any suture dehiscence, and the only fistula was located on the suture line, but was covered by the stent and not clinically relevant. The endoscopic technique is safe in its realization, including a pretty quick learning curve. The only complication attributable to the endoscopic part was the opening of the esophagus with the Hook-knife because that gesture was blinded, and was responsible for a pleural wound in 1 case. However, the technique has been demonstrated safer by using a needle puncture and hydraulic dilatation over a wire. With this adaptation, no further endoscopic complications were described in our study.

\section{Conclusion}

In conclusion, endoscopic achievement of an esophagogastric anastomosis with LAS is feasible and reproducible, without anastomotic leakage of the endoscopic suture, as part of a hybrid approach (surgical and endoscopic). Of course these preliminary results need to be confirmed in another study with improvement in perioperative management. Furthermore, risk of anastomotic stricture still needs to assessed, thus we intend to lead a study to confirm our preliminary results and address this question.

\section{Competing interests}

None

\section{References}

[1] Mariette C, Triboulet JP. [Complications following oesophagectomy: mechanism, detection, treatment and prevention]. J Chir (Paris) 2005; $142: 348-354$
[2] Watson DI, Davies N, Jamieson GG. Totally endoscopic Ivor Lewis esophagectomy. Surg Endosc 1999; 13: 293-297

[3] Bailey SH, Bull DA, Harpole DH et al. Outcomes after esophagectomy: a ten-year prospective cohort. Ann Thorac Surg 2003; 75: 217-222; discussion 222

[4] Luketich JD, Alvelo-Rivera M, Buenaventura PO et al. Minimally invasive esophagectomy: outcomes in 222 patients. Ann Surg 2003; 238: 486-94; discussion 494-495

[5] Vanbiervliet G, Bonin EA, Garcès R et al. Gastrojejunal anastomosis using a tissue-apposing stent: a safety and feasibility study in live pigs. Endoscopy 2014; 46: $871-877$

[6] Binmoeller KF, Shah J. A novel lumen-apposing stent for transluminal drainage of nonadherent extraintestinal fluid collections. Endoscopy 2011; 43: 337-342

[7] Sarela Al, Tolan DJ, Harris K et al. Anastomotic leakage after esophagectomy for cancer: a mortality-free experience. J Am Coll Surg 2008; 206: $516-523$

[8] Alanezi K, Urschel JD. Mortality secondary to esophageal anastomotic leak. Ann Thorac Cardiovasc Surg Off J Assoc Thorac Cardiovasc Surg Asia 2004; 10: $71-75$

[9] Markar SR, Karthikesalingam A, Thrumurthy S et al. Volume-outcome relationship in surgery for esophageal malignancy: systematic review and meta-analysis 2000-2011. J Gastrointest Surg Off J Soc Surg Aliment Tract 2012; 16: 1055-1063

[10] Biere SSAY, van Berge Henegouwen MI, Maas KW et al. Minimally invasive versus open oesophagectomy for patients with oesophageal cancer: a multicentre, open-label, randomised controlled trial. Lancet Lond Engl 2012; 379: 1887-1892

[11] Biere SSAY, Cuesta MA, van der Peet DL. Minimally invasive versus open esophagectomy for cancer: a systematic review and meta-analysis. Minerva Chir 2009; 64: 121-133

[12] Sihag S, Kosinski AS, Gaissert HA et al. Minimally Invasive Versus Open Esophagectomy for Esophageal Cancer: A Comparison of Early Surgical Outcomes From The Society of Thoracic Surgeons National Database. Ann Thorac Surg 2015: DOI: 10.1016/j.athoracsur.2015.09.095

[13] Kassis ES, Kosinski AS, Ross P et al. Predictors of anastomotic leak after esophagectomy: an analysis of the society of thoracic surgeons general thoracic database. Ann Thorac Surg 2013; 96: 1919-1926

[14] Wright CD, Kucharczuk JC, O'Brien SM et al. Predictors of major morbidity and mortality after esophagectomy for esophageal cancer: a Society of Thoracic Surgeons General Thoracic Surgery Database risk adjustment model. J Thorac Cardiovasc Surg 2009; 137: 587- 595; discussion 596

[15] Maas KW, Cuesta MA, van Berge Henegouwen MI et al. Quality of Life and Late Complications After Minimally Invasive Compared to Open Esophagectomy: Results of a Randomized Trial. World J Surg 2015; 39: $1986-1993$

[16] Traverso LW, Shinchi H, Low DE. Useful benchmarks to evaluate outcomes after esophagectomy and pancreaticoduodenectomy. Am J Surg 2004; 187: 604-608

[17] Price TN, Nichols FC, Harmsen WS et al. A comprehensive review of anastomotic technique in 432 esophagectomies. Ann Thorac Surg 2013; 95: 1154 - 1160; discussion 1160-1161

[18] Low DE. Evolution in surgical management of esophageal cancer. Dig Dis Basel Switz 2013; 31: 21-29

[19] Low DE, Bodnar A. Update on clinical impact, documentation, and management of complications associated with esophagectomy. Thorac Surg Clin 2013; 23: 535-550

[20] Kim RH, Takabe K. Methods of esophagogastric anastomoses following esophagectomy for cancer: A systematic review. J Surg Oncol 2010; 101: $527-533$ 
[21] Glatz T, Marjanovic G, Zirlik K et al. [Surgical treatment of esophageal cancer: Evolution of management and prognosis over the last $3 \mathrm{dec}$ ades]. Chir Z Für Alle Geb Oper Medizen 2015; 86: 662 -669

[22] von Renteln D, Vassiliou MC, McKenna D et al. Endoscopic vs. laparoscopic gastrojejunostomy for duodenal obstruction: a randomized study in a porcine model. Endoscopy 2012; 44: 161-168

[23] Vanbiervliet G, Gonzalez J-M, Bonin EA et al. Gastrojejunal Anastomosis Exclusively Using the "NOTES" Technique in Live Pigs: A Feasibility and Reliability Study. Surg Innov 2014; 21: 409-418
[24] Gonzalez J-M, Bonin EA, Vanbiervliet G et al. Evaluation of feasibility, efficiency and safety of a pure NOTES gastrojejunal bypass with gastric outlet obstruction, in an in vivo porcine model. Endosc Int Open 2013; $1: 31-38$

[25] Barthet M, Binmoeller KF, Vanbiervliet G et al. Natural orifice transluminal endoscopic surgery gastroenterostomy with a biflanged lumen-apposing stent: first clinical experience (with videos). Gastrointest Endosc 2015; 81: 215-218

\section{CORRECTION}

Adrian Culetto, Jean-Michel Gonzalez, Geoffroy Vanbiervliet et al. Endoscopic esophagogastric anastomosis with luminal apposition Axios stent (LAS) approach: a new concept for hybrid "Lewis Santy".

Endoscopy International Open 2017; 05: E455 - E462

DOI: 10.1055/s-0043-106577

The name of the coauthor Pablo Miranda Garcia was wrong. Correct is: Pablo Miranda Garcia. This was corrected in the online version on January 10, 2018. 